\title{
Lysyl oxidase assists tumor-initiating cells to enhance angiogenesis in hepatocellular carcinoma
}

\author{
MIN YANG ${ }^{1 *}$, JINGTAO LIU $^{2 *}$, FEI WANG $^{3}$, ZHIHUA TIAN $^{4}$, \\ BO MA ${ }^{5}$, ZHONGWU LI ${ }^{6}$, BOQING WANG ${ }^{7}$ and WEI ZHAO ${ }^{8}$
}

\begin{abstract}
${ }^{1}$ Department of Gerontology, Beijing Shijitan Hospital, Capital Medical University, Beijing 100038;
${ }^{2}$ Key Laboratory of Carcinogenesis and Translational Research (Ministry of Education), Department of Pharmacy, Peking University Cancer Hospital and Institute, Beijing 100142, P.R. China; ${ }^{3}$ Division of Pediatrics, University of Texas M.D. Anderson Cancer Center, Unit 0853, Houston, TX 77030, USA; ${ }^{4}$ Key Laboratory of Carcinogenesis and Translational Research (Ministry of Education), Department of Central Laboratory; ${ }^{5}$ Key Laboratory of Carcinogenesis and Translational Research (Ministry of Education), Department of Lymphoma; ${ }^{6} \mathrm{Key}$ Laboratory of Carcinogenesis and Translational Research (Ministry of Education), Department of Pathology, Peking University Cancer Hospital and Institute, Beijing 100142; ${ }^{7}$ Department of Hepatopancreatobiliary Surgery, Affiliated Tumor Hospital of Xinjiang Medical University, Urumqi, Xinjiang 830011; ${ }^{8}$ Key Laboratory of Carcinogenesis and Translational Research (Ministry of Education), Department of Cell Biology, Peking University Cancer Hospital and Institute, Beijing 100142, P.R. China
\end{abstract}

Received August 7, 2018; Accepted December 14, 2018

DOI: 10.3892/ijo.2019.4705

\begin{abstract}
A highly tumorigenic and malignant sub-population of HCC containing tumor-initiating cells (TICs) are defined by high self-renewal and sphere formation ability. Lysyl oxidase (LOX) regulates various factors involved in extracellular matrix (ECM) maintenance, migration and angiogenesis. Certain reports have demonstrated the role of LOX in ECM crosslinking, however, the cancer-promoting effects of LOX in HCC remain unclear, and whether LOX has a role in the regulation of angiogenesis in HCC TICs has not been elucidated. In the current study, RNA sequencing using next-generation sequencing technology and bioinformatics analyses revealed that LOX gene expression was significantly upregulated in cell spheres. Sphere cells may form tumors with more vascular
\end{abstract}

Correspondence to: Dr Wei Zhao, Key Laboratory of Carcinogenesis and Translational Research (Ministry of Education), Department of Cell Biology, Peking University Cancer Hospital and Institute, Room 603, Scientific Research Building, 52 Fucheng Road, Haidian, Beijing 100142, P.R. China

E-mail: linelong@126.com

Dr Boqing Wang, Department of Hepatopancreatobiliary Surgery, Affiliated Tumor Hospital of Xinjiang Medical University, 789 Suzhou East Street, Xinshi, Urumqi, Xinjiang 830011, P.R. China

E-mail: bqwanghps@163.com

*Contributed equally

Key words: hepatocellular carcinoma, tumor-initiating, lysyl oxidase, angiogenesis, sorafenib enrichment compared with tumors produced from adherent cells, as observed in a mouse xenograft model. LOX expression is correlated with increased vascular endothelial growth factor (VEGF) and platelet-derived growth factor, as demonstrated by analyses of The Cancer Genome Atlas and Gene Expression Omnibus databases. Conditioned media obtained from LOX-overexpressing tumor cells stimulated angiogenesis via secreted VEGF and enhanced the tube formation capacity of endothelial cells. Furthermore, these functional behaviors were blocked by the LOX inhibitor $\beta$-aminopropionitrile. These findings provide novel mechanistic insight into the pivotal role of LOX in the regulation of TICs in HCC. Combination of LOX inhibitor with sorafenib is a potentially advantageous strategy for HCC therapy.

\section{Introduction}

Hepatocellular carcinoma (HCC) is one of the deadliest malignancies worldwide. It is also the third most common cancer in males (accounting for $12.72 \%$ of cancer incidence) and the fifth most common in females (accounting for $5.68 \%$ of cancer incidence) in China. The mortality rate of $\mathrm{HCC}$ is $\sim 25 \%$, primarily due to a high rate of recurrence (1). Chemotherapy drugs can effectively control the progression of HCC. However, tumor cells that are insensitive to these drugs remain present in the liver, which may subsequently lead to HCC recurrence (2). These cells may belong to a stem cell-like population known as tumor-initiating cells (TICs) (3). Although stem cell-like properties, including self-renewal, tumorigenicity and dedifferentiation, are closely associated with secondary tumor growth in HCC (4), the contribution of TICs to tumor angiogenesis has not been elucidated.

Growing evidence suggests an association between TICs and angiogenesis (5). Previous studies have reported that 
CD24(+) tumorigenic cells, which are involved in initiating, maintaining and expanding tumor growth, have TIC properties and angiogenic potential (6). As a marker of angiogenic endothelial cells, CD13 has recently been indicated to be a biomarker of human liver cancer stem cells, and a fusion protein composed of CD13-targeting peptide Asn-Gly-Arg was able to reduce endothelial tube formation (7). CD133(+) cancer stem cells exhibit increased tumor-initiating potential and tumor-endothelial cell interaction (8). In a HCC xenograft mouse model, low-dose metronomic gemcitabine significantly slowed tumor growth, and decreased levels of cancer stem-like cells and epithelial progenitor cells, which are dependent on the vascular microenvironment (9). These cancer stem-like cells or TIC-enriched cells of HCC are identified by different markers, including Kruppel like factor 5 (KLF5) (10), SRY-box 9 (SOX9) (11), SOX12 (12) and aldehyde dehydrogenase (13), and have similar characteristics, namely, self-renewal, sphere formation and long-term growth with no differentiation. This sphere-derived population of stem-like cells has been widely used to investigate TICs $(13,14)$, as the spheroid culturing of cancer and stromal cells promotes their stem-like properties and accelerates the density of vascular formations (15).

A secreted enzyme, lysyl oxidase (LOX), reportedly contributes to tumor angiogenesis $(16,17)$. Vascular endothelial growth factor (VEGF) results in phosphorylation of AKT, ERK and JNK pathways and active p65, which was demonstrated to be inhibited by LOX silencing (18). LOX has been reported to be a predictor of less favorable outcomes and may regulate the expression of VEGF via p38 mitogen-activated protein kinase (MAPK) signaling (19). It has also been reported modulating the expression of heat shock-induced factor- $1 \alpha$ $(\mathrm{HIF}-1 \alpha) / \mathrm{LOX}$ pathway attenuates the metastatic potential of liver cancer stem cells (20). Therefore, the importance of angiogenesis in cancer development and recurrence is well established, as an adequate blood supply is essential for tumor growth dependent or independent of TIC properties. Additionally, LOX is involved in the mechanistic target of rapamycin kinase (21), transforming growth factor- $\beta$ (22) and phosphatidylinositol 3-kinase (PI3K) (23) signaling axis, mediates collagen-linking in tumor microenvironments, and associated with the activity of integrin $\beta 1$ (24) and CD44 (25). Notably, HIF-1 $\alpha$, integrin $\beta 1$ and CD44 have essential roles in embryonic stem cells and TICs. Thus, LOX may be required to create a permissive niche environment for tumorigenesis, with particular relevance for TICs. Consequently, LOX may be a potential therapeutic target for $\mathrm{HCC}$ via effects on TICs and angiogenesis (19).

In the current study, it was identified that LOX has a bridge role of stem cell-like properties, acting to promote angiogenesis upon endothelial cell (EC) proliferation and tube formation. Furthermore, LOX, which is secreted from TICs, enhanced angiogenesis via activation of the VEGF signaling pathway. Furthermore, anti-angiogenesis therapy is a general strategy against $\operatorname{HCC}(26,27)$, such as using sorafenib $(28,29)$ or rageferinib $(30)$. The effect of combination therapy of a LOX inhibitor and sorafenib on EC proliferation was determined. Considering all of the above, the findings suggest that TICs secrete LOX to promote tumor angiogenesis, which is blocked by sorafenib in combination with a LOX inhibitor.

\section{Materials and methods}

Cell lines and culture conditions. Human umbilical vein endothelial cells (hUVECs) were obtained from the cell bank at the Chinese Academy of Sciences (Shanghai, China). The HEP cell line was established from patient HCC tissue from Peking University Cancer Hospital (PUCH; Beijing, China), as previously described (31). The HCC cell line HuH-7 (cat. no. JCRB0403) was purchased from the Health Science Research Resources Bank (Osaka, Japan). Cells were cultured in RPMI-1640 medium (Invitrogen; Thermo Fisher Scientific, Inc., Waltham, MA, USA) supplemented with $10 \%$ fetal bovine serum (FBS; cat. no. 10099-141; Invitrogen; Thermo Fisher Scientific, Inc.) and $1 \%$ penicillin/streptomycin (Invitrogen) at $37^{\circ} \mathrm{C}$ in a humidified incubator under an atmosphere of $95 \%$ air plus $5 \% \mathrm{CO}_{2}$. After culture for $48 \mathrm{~h}$ with in conditioned medium (CD CHO medium; cat. no. 10743011; FBS-free, $1 \%$ penicillin/streptomycin; Invitrogen; Thermo Fisher Scientific, Inc.), the supernatant from each group of cells was harvested and concentrated 10-fold using a Millipore concentration tube (10 kDa nominal molecular weight limit; EMD Millipore, Billerica, MA, USA) according to the manufacturer's protocol.

HCC tissue was obtained from a 47-old male patient that underwent $\mathrm{HCC}$ resection of his right-half liver surgery at PUCH in February 2004. The use was approved by the Ethics Committee of PUCH (no. 2014KT-09). All studies involving human samples adhered to the principles of the Declaration of Helsinki in accordance with the National Institutes of Health guidelines with patient consent (31).

Sequencing andbioinformationanalysis. Total RNAs extracted from cultured cells using TRIzol reagent (Invitrogen; Thermo Fisher Scientific, Inc.) were sequenced by the Illumina Hiseq X10 platform (Illumina, Inc., San Diego, CA, USA) to obtain raw reads. Filtered clean reads had equality distributed base composition and mass. The quality of sequencing outcomes was subjected to subsequent bioinformatics analysis. The upregulated differentially expressed genes was clustered by Kyoto Encyclopedia of Genes and Genomes (KEGG) pathway enrichment analysis, which performed using the Database for Annotation, Visualization and Integrated Discovery (DAVID; david.ncifcrf.gov/). The protein-protein interaction network of upregulated differentially expressed genes was constructed using the Search Tool for the Retrieval of Interacting Genes database (string-db.org/) followed by highest confidence (score, $>0.9$ ). High relational proteins were analyzed and illustrated with biological pathway using FunRich software version 3 (funrich.org/). The mRNA-sequencing expression profiles and clinical data from HCC samples were downloaded from The Cancer Genome Atlas (TCGA; cancergenome.nih. gov/), and then analyzed gene transcript per million between normal and tumor tissues using web resource UALCAN (ualcan.path.uab.edu.) which access to publicly available cancer transcriptome data.

Sphere formation. Each well contained 6,000 single cells that were plated in Ultra Low Attachment 6-well plates (Corning Life Science, Acton, MA, USA) and cultured in $2 \mathrm{ml} \mathrm{1:1} \mathrm{mix} \mathrm{of}$ $2 \%$ methylcellulose (Sigma-Aldrich; Merck KGaA, Darmstadt, 
Table I. Information for antibodies used in WB and IHC.

\begin{tabular}{llccc}
\hline Name & \multicolumn{1}{c}{ Supplier } & Catalogue number & Species & Dilution \\
\hline$\alpha 2 \delta 1$ & Abcam & ab2864 & Mouse monoclonal IgG & WB 1:2,000 \\
LOX & Abcam & ab31238 & Rabbit polyclonal IgG & WB 1:2,000 \\
Nanog & Abcam & ab109250 & Rabbit monoclonal IgG & WB 1:2,000 \\
OCT4 & Abcam & ab18976 & Rabbit polyclonal IgG & WB 1:2,000 \\
SOX2 & Epitomics; Abcam & ab97959 & Rabbit polyclonal IgG & WB 1:2,000 \\
VEGF & Abcam & ab53465 & Rabbit polyclonal IgG & WB 1:2,000; IHC 1:200 \\
CD105 & Abcam & ab11414 & Mouse monoclonal IgG & IHC 1:200 \\
ERK1/2 & Cell Signaling Technology, Inc. & 9102 & Rabbit polyclonal IgG & WB 1:2,000 \\
p-ERK1/2 & Cell Signaling Technology, Inc. & 9101 & Rabbit polyclonal IgG & WB 1:2,000 \\
Actin & Roche Diagnostics & 1378996 & Mouse monoclonal IgG & WB 1:10,000 \\
\hline
\end{tabular}

WB, western blot; IHC, immunohistochemistry; $\alpha 2 \delta 1$, voltage-dependent calcium channel subunit $\alpha 2 \delta 1$; LOX, lysyl oxidase; OCT4, POU domain, class 5 transcription factor 1; SOX2, SRY-box 2; VEGF, vascular endothelial growth factor; ERK1/2, extracellular signal-regulated kinase 1/2; p-ERK1/2, phospho-ERK1/2.

Germany) and Dulbecco's modified Eagle's medium/F12 (Invitrogen; Thermo Fisher Scientific, Inc.) supplemented with B27 (1:50), $20 \mathrm{ng} / \mathrm{ml}$ epidermal growth factor, $20 \mathrm{ng} / \mathrm{ml}$ basic fibroblast growth factor and $10 \mathrm{ng} / \mathrm{ml}$ hepatocyte growth factor (Invitrogen). The spheres over $100 \mu \mathrm{m}$ in diameter were harvested under a stereomicroscope (Olympus Corporation, Tokyo, Japan) following incubation at $37^{\circ} \mathrm{C}$ and in $5 \% \mathrm{CO}_{2}$ for 2-3 weeks. The cell lines induced to form spheres were termed 'HEP-sph' and 'HuH-7-sph', compared with the parental control.

Transfection of LOX expression plasmid and identification. A lentivirus overexpressing LOX was constructed using the pLenti6-Blast lentivirus vector containing a full long cDNA clone of LOX from GeneCards (GCID GC05M122063) using restriction endonuclease Bam $\mathrm{H} 1$ and Xhol (New England BioLabs, Inc., Ipswich, MA, USA). Lentiviral packaging was performed by transfecting 106 293FT cells with each $3 \mu \mathrm{g}$ plasmid of the ViraPower Packaging Mix (Invitrogen; Thermo Fisher Scientific, Inc.) using Lipofectamine ${ }^{\circledR} 2000$. HEP and HuH-7 cells were infected using recombinant lentiviruses and vector empty control, respectively (10 multiplicity of infection), and selected stably infected cells by $5 \mu \mathrm{g} / \mathrm{ml}$ blasticidin resistance according to manufacturer's protocol as previously described (32). The cell lines infected with LOX and the vector control were termed 'HEP-LOX' and 'HuH-7-LOX'; the controls were HEP-C and HuH-7-C. LOX mRNA and protein levels were analyzed using reverse transcription-quantitative polymerase chain reaction (RT-qPCR) and western blot analysis.

Western blot analysis. Cells were suspended in an appropriate volume of lysis buffer (50 mM Tris $\mathrm{pH} 7.4,150 \mathrm{mM} \mathrm{NaCl}$, $1 \%$ NP-40, $0.25 \%$ sodium deoxycholate and $0.1 \%$ SDS) supplemented with $1 \mathrm{mM}$ phenylmethylsulfonyl fluoride, phosphatase inhibitor cocktail and Complete Mini Protease Inhibitor Cocktail (Roche Diagnostics GmbH, Mannheim, Germany). Following protein quantitation using bicinchoninic acid assay, $30 \mathrm{mg}$ HEP, HEP-sph, HuH-7, and HuH-7-sph cells protein lysate were resolved by SDS-PAGE on $10 \%$ gels and electroblotted onto nitrocellulose membranes. Following blocking using 5\% fat-free milk at room temperature for $1 \mathrm{~h}$, the membranes were incubated with primary antibodies specific to LOX, VEGF, CD105, phospho (p)-extracellular signal-regulated kinase (ERK), voltage-dependent calcium channel subunit $\alpha 2 \delta 1(\alpha 2 \delta 1)$, Nanog, POU domain, class 5 transcription factor 1 (OCT4), SOX2 and actin (Table I) at room temperature for $1 \mathrm{~h}$, and corresponding secondary horseradish peroxidase (HRP)-conjugated goat anti-rabbit (cat. no. 111-035-003) or anti-mouse antibodies (cat. no. 115-035-003; dilution 1:50,000; Jackson ImmunoResearch Laboratories, Inc., West Grove, PA, USA) at room temperature for $1 \mathrm{~h}$. Immobilon ${ }^{\mathrm{TM}}$ Western Chemiluminescent HRP substrate (Merck KGaA, Darmstadt, Germany) was used to detect the immuno-complexes.

$R T-q P C R$ analysis. Total RNA was extracted from HEP, HEP-sph, HuH-7 and HuH-7-sph cells using TRIzol reagent (Invitrogen; Thermo Fisher Scientific, Inc.), and cDNA was subsequently synthesized from $2 \mu \mathrm{g}$ total RNA using the MultiScribe ${ }^{\mathrm{TM}}$ Reverse Transcriptase at $37^{\circ} \mathrm{C}$ for $50 \mathrm{~min}$ (Invitrogen; Thermo Fisher Scientific, Inc.). RT-qPCR was performed on an ABI7500 PCR machine using the SYBR Green PCR Master Mix (Toyobo Life Science, Osaka, Japan) and specific primers for each gene were designed and validated by using PrimerDepot (primerdepot.nci.nih.gov; Table II). GAPDH was used as an internal control. PCR using 40 cycles of $95^{\circ} \mathrm{C}$ for $15 \mathrm{sec}$ and $60^{\circ} \mathrm{C}$ for $1 \mathrm{~min}$ was performed in duplicate. The relative gene expression for each sample was calculated using the formula $2^{(-\Delta \mathrm{Cq})}=2^{[\mathrm{Cq}(\mathrm{GAPDH})-\mathrm{Cq}(\operatorname{target})]}(33)$.

LOX activity assay. LOX activity in the culture medium from HCC cells was measured using an Amplite ${ }^{\mathrm{TM}}$ Fluorimetric Lysyl Oxidase Assay kit (AAT Bioquest Inc., Sunnyvale, CA, USA) following the protocols provided by the manufacturers as described as reported (34).

ELISA. LOX secretion was measured by ELISA (eBioscience; Thermo Fisher Scientific, Inc.), according to the manufacturer's 
Table II. Primer sequences.

\begin{tabular}{lll}
\hline Gene & \multicolumn{1}{c}{ Sense } & \multicolumn{1}{c}{ Anti-sense } \\
\hline BMI1 & 5'-AGCAGCAATGACTGTGATGC-3' & 5'-CAGTCTCAGGTATCAACCAG-3' \\
KLF4 & 5'-AAGCCAAAGAGGGGAAGAC-3' & 5'-CATCTGAGCGGGCGAATTTC-3' \\
LOX & 5'-GTTCCAAGCTGGCTACTC-3' & 5'-GGGTTGTCGTCAGATAC-3' $^{\prime}$ \\
Nanog & 5'-TGCCTCACACGGAGACTGTC-3' & 5'-TGCTATTCTTCGGCCAGTTG-3' \\
OCT-4 & 5'-GACAACAATGAAAATCTTCAGGAGA-3' & 5'-CTGGCGCCGGTTACAGAACCA-3' \\
SOX2 & 5'-ACATGAACGGCTGGAGCAAC-3' & 5'-AGGAAGAGGTAACCACAGGG-3' \\
VEGF & 5'-CTACCTCCACCATGCCAAGT-3' & 5'-AGCTGCGCTGATAGACATCC-3'
\end{tabular}

BMI1, BMI1 proto-oncogene, polycomb ring finger; KLF4, Kruppel like factor 4; LOX, lysyl oxidase; OCT4, POU domain, class 5 transcription factor 1; SOX2, SRY-box 2; VEGF, vascular endothelial growth factor.

instructions (35). Cell culture supernatant samples were collected following the periods of incubation with HEP-C, HEP-LOX, HuH-7-C and HuH-7-LOX cultured with or without $100 \mu \mathrm{mol} / 1 \beta$-aminopropionitrile (BAPN). Standards and samples were added to the appropriate microtiter plate wells, which were pre-coated with an antibody specific to LOX. Following addition of reagents to every well and incubation at $37^{\circ} \mathrm{C}$ for $2 \mathrm{~h}$, a color change was detected using iMark ${ }^{\mathrm{TM}}$ microplate reader (BioRad Laboratories, Inc., Waltham, MA, USA) at a wavelength of $450 \pm 2 \mathrm{~nm}$. The concentration of LOX in every sample was determined by comparing the optical density of the samples to a standard curve.

Cell proliferation assay. Aliquots of $1 \times 10^{5}$ cells/ml hUVECs were seeded in a 96 -well microplate. After incubation at $37^{\circ} \mathrm{C}$ under $5 \% \mathrm{CO}_{2}$ for $24 \mathrm{~h}$, cells were treated with the culture supernatant of HEP, HEP-sph, HuH-7, and HuH-7-sph cells, with or without BAPN for further $48 \mathrm{~h}$ cultivation using no-phenol-red CHO medium (Invitrogen; Thermo Fisher Scientific, Inc.). Then, cells were exposed to MTS method using CellTiter 96 one solution cell proliferation assay kit (Promega Corporation, Madison, WI, USA) for $2 \mathrm{~h}$ and measured spectrophotometrically at a wavelength of $450 \pm 2 \mathrm{~nm}$.

Tube formation assay. The tube formation assay was performed as previously described (36). Initially, $4 \mathrm{mg} / \mathrm{ml}$ Matrigel dissolved in PBS was added into 96-well plates $(50 \mu \mathrm{l} /$ well $)$ and the plates were incubated at $37^{\circ} \mathrm{C}$ for $30 \mathrm{~min}$ to allow gel formation. Subsequently, $1 \times 10^{5}$ cells/ml hUVECs in the logarithmic growth phase were starved and plated into each well with medium containing the culture supernatant of HEP, HEP-sph, HuH-7 and HuH7-sph cells with or without BAPN. After incubation for $72 \mathrm{~h}$, six randomly chosen fields per well were captured, the 2-dimensional organization of the cells was imaged using IncuCyte ${ }^{\circledR}$ image observation (Essen Bioscience, Ann Arbor, MI, USA), and the network growth area of blood vessels was calculated using ImageJ v1.41 (rsb. info.nih.gov/ij/) (37).

Experimental proliferation on chick chorioallantoic membrane assay (CAM). Ten-day-old chicken eggs obtained from Vital River Laboratories Co., Ltd. (Beijing, China) following fertilization were windowed in the shell over a chorioallantoic membrane vein. Briefly, the area of the outer eggshell where prominent blood vessels are located close to the inner shell surface was swabbed with $70 \%$ ethanol and a small window was cut through the eggshell with a hobby-grinding wheel. Cells $\left(5 \times 10^{6}\right)$ in a total volume of $30 \mu 1$ RPMI-1640 medium were transplanted gently to the CAM and reagents covered the surface of the tumor following the xenograft for $24 \mathrm{~h}$. The window was sealed using sterile adhesive plaster, and the embryo was allowed to develop for an additional 7 days in a humidified forced-draft egg incubator $\left(37^{\circ} \mathrm{C}\right)$. The embryo was then sacrificed. Tumors growing on the CAM were dissected and weighed to evaluate growth rate in vivo.

Animal samples and immunofluorescence. In order to establish a tumor-bearing mouse model, HEP, HEP-sph, HuH-7 and HuH-7-sph cells were mixed with $100 \mu$ l equal volume plain RPMI-1640 and Matrigel (10 mg/ml; BD Biosciences; Becton, Dickinson and Company, Franklin, Lakes, NJ, USA). The cell suspension was subcutaneously injected into the back of six female 4-6 week old NOD/SCID mice (18 g; Vital River Laboratories Co., Ltd.). Animals were housed under individual ventilated cages system at $22 \pm 2^{\circ} \mathrm{C}$ and $40-60 \%$ atmosphere with alternating $12 \mathrm{~h} \mathrm{light/dark} \mathrm{cycle.} \mathrm{Food} \mathrm{and} \mathrm{sterile} \mathrm{water}$ was freely available and changed every three days. Tumor formation was monitored every week. At 10 weeks later, tumors were frozen immediately in liquid nitrogen and stored at $-80^{\circ} \mathrm{C}$. All animal experiments were approved by and conformed to the regulatory standards of Peking University Cancer Hospital on Laboratory Animals Care and Use in accordance with the National Institutes of Health Guide (Guide for the Care and Use of Laboratory Animals) (38). For immunofluorescence staining, frozen tumor tissues were sectioned (4- $\mu$ m-thick) using a cryostat and fixed in methanol for 30-40 sec. Following blocking with 5\% non-fat milk in PBS at room temperature for $1 \mathrm{~h}$, slides were incubated with an antibody specific to LOX at $4^{\circ} \mathrm{C}$ overnight, followed by a reaction with fluorescein isothiocyanate-goat-anti-rabbit IgG. Nuclei were stained with DAPI at $0.5 \mu \mathrm{g} / \mathrm{ml}$ at room temperature for $5 \mathrm{~min}$. All the specimens were mounted in $90 \%$ glycerol/PBS with 2.5\% 1,4-diazabicyclo $(2,2,2)$ octane and examined using a Leica SP5 confocal microscope (Leica Microsystems GmbH, Wetzlar, Germany). For hematoxylin and eosin (H\&E) staining, the tissues were fixed in 
A
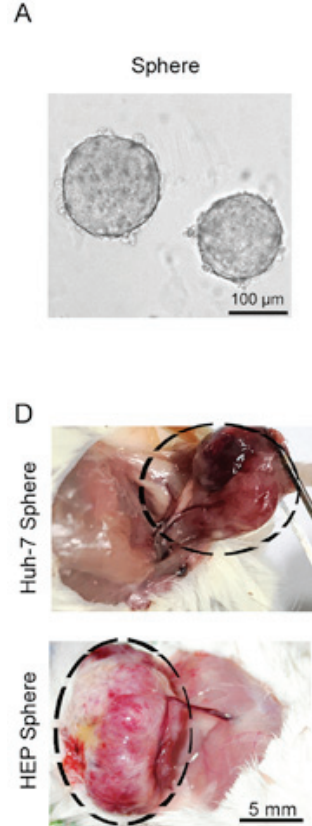

$F$

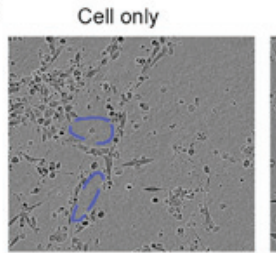

B

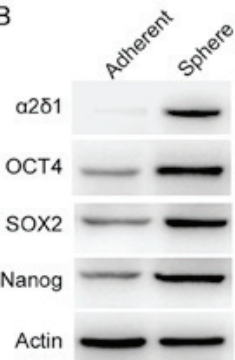

E VEGF
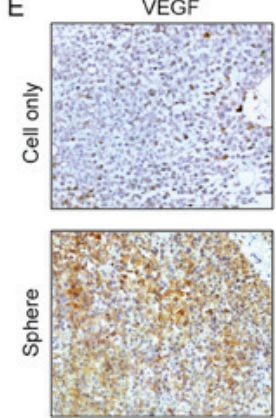

Control $\mathrm{CM}$

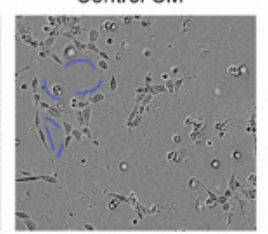

C

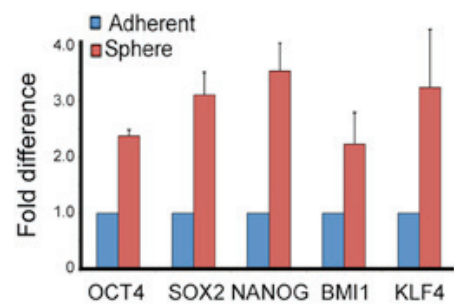

CD105

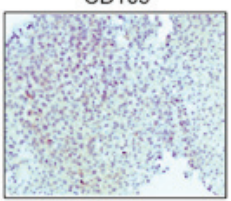

H\&E
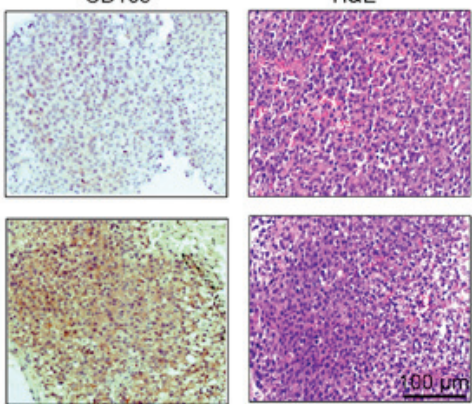

Sphere CM
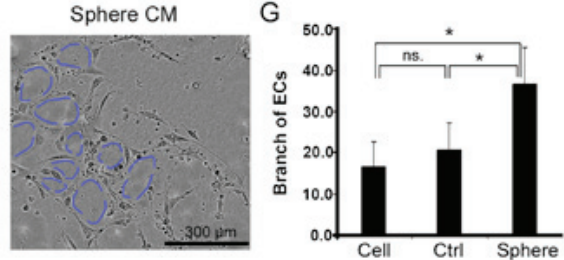

Figure 1. Sphere-derived cells contribute to HCC angiogenesis. (A) Sphere formation of cells is performed using serum-free medium supplied with B27/epidermal growth factor/fibroblast growth factor. (B) Stem-associated protein was validated by western blot and (C) RNA levels are detected by reverse transcription-quantitative polymerase chain reaction. Transplanted spheres into NOD/SCID mice. (D) A large number of vessels and capillaries are available surrounding tumor tissues, which grow up from sphere-derived cells of HuH-7 (maximum tumor diameter $1.32 \mathrm{~cm}$ ) and HEP (maximum tumor diameter $1.67 \mathrm{~cm}$ ). (E) Immunohistochemical staining of VEGF and CD105 are performed on tumor slides form HuH-7 cell and sphere derived transplantations, and histopathological H\&E staining. (F) Tube formation of human ECs using CM from Huh-7 sphere-derived cells. (G) Measurement of EC tube branches. ${ }^{*} \mathrm{P}<0.05$ using one-way analysis of variance. $\alpha 2 \delta 1$, voltage-dependent calcium channel subunit $\alpha 2 \delta 1$; OCT4, POU domain, class 5 transcription factor 1 ; SOX2, SRY-box 2; BMI1, BMI1 proto-oncogene, polycomb ring finger; KLF4, Kruppel like factor 4; VEGF, vascular endothelial growth factor; H\&E, hematoxylin and eosin; CM, conditioned media; ECs, endothelial cells; ns, not significant.

$4 \%$ paraformaldehyde and dehydrated through a serial alcohol gradient, and embedded in paraffin wax blocks. following sectioning of 5- $\mu \mathrm{m}$-thick slides and rehydration in xylene and ethanol, H\&E were used to stain the tissues.

Statisticalanalysis. Experiments were performed independently in triplicate, and data are presented as the mean \pm standard deviation. Statistical significance was determined using the unpaired two-sided Student's t-test for independent samples with Excel 2010 software (Microsoft Corporation, Redmond, WA, USA). In case of multiple tests, one-way analysis of variance followed by Tukey's multiple comparison test was applied. Correlation was performed using two-tailed Spearman's test using GraphPad Prism 5.0 version (GraphPad Software, Inc., La Jolla, CA, USA). P $<0.05$ was considered to indicate a statistically significant difference.

\section{Results}

TIC-enriched cells contribute to HCC angiogenesis. TIC-enriched cells drive tumor formation and processing because of their stem cell characteristics (4) and, in particular, their capacity for self-renewal. Sphere cells are a group of stem-like cells, as previously reported and shown in Fig. 1A. The expression of a panel of proteins associated with TICs, including OCT4, Nanog, SOX2 and novel liver cancer stem cell marker $\alpha 2 \delta 1$, was validated by western blot (Fig. 1B) and RT-qPCR (Fig. 1C). In a mouse model, a large number of vessels and capillaries surrounded tumor tissues and were deep inside xenograft tumors, which were produced from the sphere-derived cells (Fig. 1D). These properties of angiogenic morphology fundamentally distinguish TICs from tumor cells. To explore the association between TICs and tumor angiogenesis, immunohistochemical staining of CD105 and VEGF was performed on the tumor slides, and histopathological H\&E staining (Fig. 1E). Elevated expression of CD105 and VEGF was present in sphere-derived tumors. Furthermore, the CM from cultured HuH-7 sphere cells significantly increased tube formation among hUVECs (Fig. 1F). The branching of hUVEC tube networks was calculated using ImageJ software (Fig. 1G). The findings indicated that CM from culturing sphere cells induced hUVEC proliferation and tube formation. These results indicate that sphere-derived cells and their secreted substrate could directly activate angiogenesis with HCC tumorigenesis. 
A

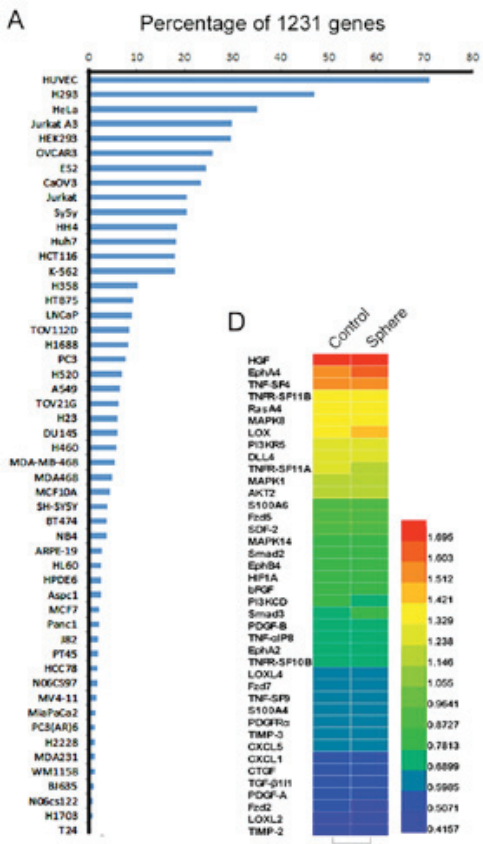

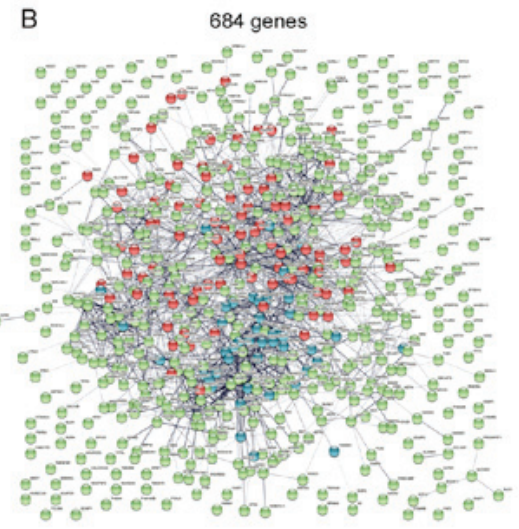

C

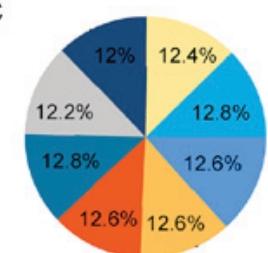

- Integrin family cell surface interaction

$=$ PDGF recepter signaling newwork

= Avf6 signaling events

- EGF receptor signaling pathway

= VEGF and VEGFR signaling network

- PDGFR-beta signaling pathway

IGF1 pathway

EGFr-dependent Endothelin signaling
events

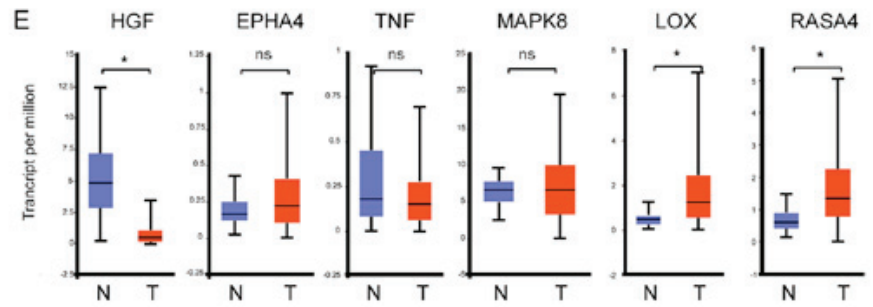

Figure 2. Sequencing and bioinformatics analysis. Sequencing analysis was performed to compare sphere-derived and Petri dish cells from patient primary cultures. (A) A total of 1,232 differentially regulated genes of HEP-sph cells were detected and Kyoto Encyclopedia of Genes and Genomes was used to cluster cell lines highly related to these genes. (B) Of the 1,232 genes, 684 were clustered by the Markov Cluster Algorithm with an inflation parameter (number: 3 ) using the STRING database with different average node degrees. Red, $>1.0$; blue, $>0.5,<1.0$; green, $<0.5$. (C) Angiogenesis response molecular signaling analysis of these 684 genes using the FunRich analysis tool. (D) The heat map presented relevant angiogenesis pathway genes and their scores among these 684 genes using Gene Set Enrichment Analysis website. (E) Some subunits of HGF, TNF, PI3K, LOX and EPHA4 were highly increased in tumors compared with their normal tissues based on The Cancer Genome Atlas data using UALCAN. $\mathrm{P}<0.05$ using a two-sided Student's t-test. PDGF, platelet-derived growth factor; Arf6, ADP ribosylation factor 6; EGF, epidermal growth factor; VEGF, vascular endothelial growth factor; VEGFR, VEGF receptor; PDGFR, platelet-derived growth factor receptor; IGF1, insulin-like growth factor 1; EGFR, EGF receptor; HGF, hepatocyte growth factor; EPHA4, ephrin type-A receptor 4; TNF, tumor necrosis factor; MAPK8, mitogen-activated protein kinase 8; LOX, lysyl oxidase; RASA4, Ras GTPase-activating protein 4.

LOX activates angiogenesis signaling by sequencing and bioinformation analysis. To identify the characteristics of TIC-enriched cell populations, sequencing analysis comparing sphere cells and non-sphere cells from patient primary culture cells was performed to confirm which genes were up- or downregulated during the angiogenic response. Notably, $>70$ percent of 1,232 differentially regulated genes were highlighted in hUVECs via analysis by KEGG, which reportedly affect cell signal pathways or functional behaviors of hUVECs (Fig. 2A). Upregulated genes were clustered for protein-protein interactions using STRING network analysis V 10.5 (Fig. 2B). A total of 684 genes were then selected by clustering interaction using the FunRich functional enrichment analysis tool (39). The majority of genes were associated with angiogenesis, such as platelet-derived growth factor (PDGF) receptors, the integrin family, the epidermal growth factor receptor, and components of the VEGF network and IGF pathway (Fig. 2C). Furthermore, a heat map of the relevant pathways of angiogenesis is shown in Fig. 2D. Some of the subunits of hepatocyte growth factor (HGF), erythropoietin producing hepatocyte A4 (EPHA4), tumor necrosis factor (TNF), PI3K and LOX were greatly increased in sphere-derived cells. Furthermore, analysis of TCGA database by UALCAN (http://ualcan.path.uab. $\mathrm{edu} /$ ) (40) revealed that the mRNA levels of LOX and Ras GTPase-activating protein 4 expression were higher in HCC tissues (370 cases) than in normal controls (50 cases), but HGF, EPHA4, TNF, MAPK8 and others were not increased.
LOX is an activator of angiogenesis, and LOX associated with $P D G F$ and VEGF. The significantly elevated LOX mRNA was identified from the Gene Expression Omnibus (GEO) profile of a PDGF-driven genetic mouse model (GDS5320:10458894; Fig. 3A). The highly upregulated LOX indicates that it may have a critical role in mediating tumor angiogenesis in a mouse model. Another GEO database with human clinical samples (GDS4887) (41) was analyzed, which revealed significant correlations between the mRNA level of LOX and VEGF were in the tumor tissues of patients with $\mathrm{HCC}(\mathrm{P}=0.0034$; Fig. 3B); however, there was no significant correlation in normal tissues $(\mathrm{P}=0.55$; Fig. $3 \mathrm{C})$. In the TCGA database, LOX and VEGFA $\left(r^{2}=0.066, P<0.001\right)$, LOX and VEGFB $\left(r^{2}=0.008, P=0.08\right)$; LOX and VEGFC $\left(\mathrm{r}^{2}=0.02, \mathrm{P}=0.0073\right)$ were significantly correlated (Fig. 3D). These results suggest that LOX may be involved in stimulating tumor angiogenesis, which is associated with sphere-derived HCC cells.

TIC-enriched HCC cells express high LOX levels. It has been reported that LOX is upregulated in tumors and their associated endothelial cells (42), but seldom in TICs. In the current study, RT-qPCR demonstrated that $L O X$ mRNA is highly expressed in sphere-derived cells, including HEP-sph and HuH7-sph cells (Fig. 4A). The protein level of extracellular secreted LOX was determined using an ELISA and western blot analysis (Fig. 4B). LOX secretion in was increased in the sphere-derived cells compared with the parental control cell lines, HuH-7 and HEP cells. Additionally, LOX secretion 

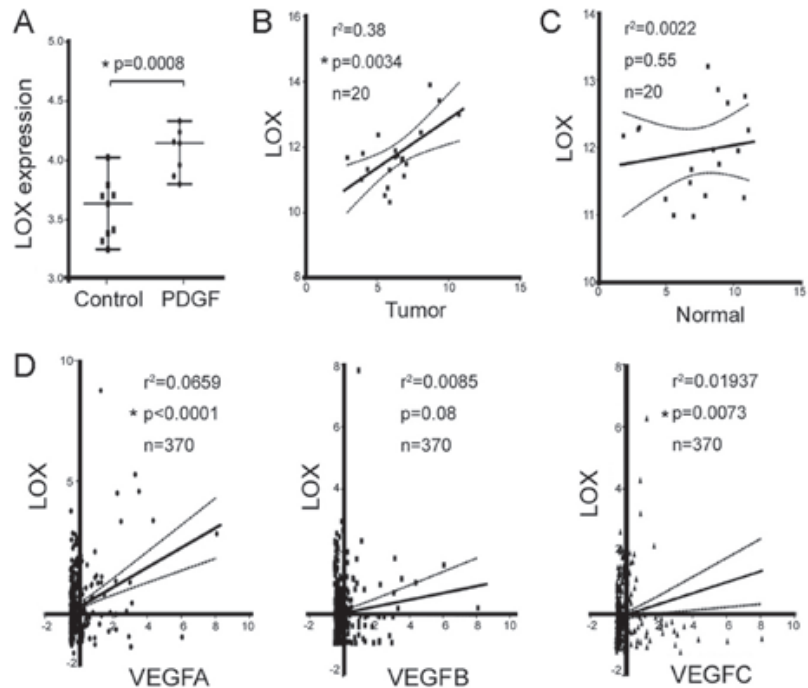

Figure 3. Association between LOX and angiogenic factors. (A) GEO profiles (GDS5320:10458894) analyzed the levels of LOX mRNA in normal and hepatocellular carcinoma tissues isolated from a genetic mouse model driven by PDGF. Correlations between LOX and VEGF in (B) tumor and (C) normal control tissues using GEO profiling (GDS4887). (D) The association between LOX and VEGF was analyzed using The Cancer Genome Atlas database. ${ }^{*} \mathrm{P}<0.05$ using a two-tailed Spearman's test. GEO, Gene Expression Omnibus; LOX, lysyl oxidase; PDGF, platelet-derived growth factor; VEGF, vascular endothelial growth factor.
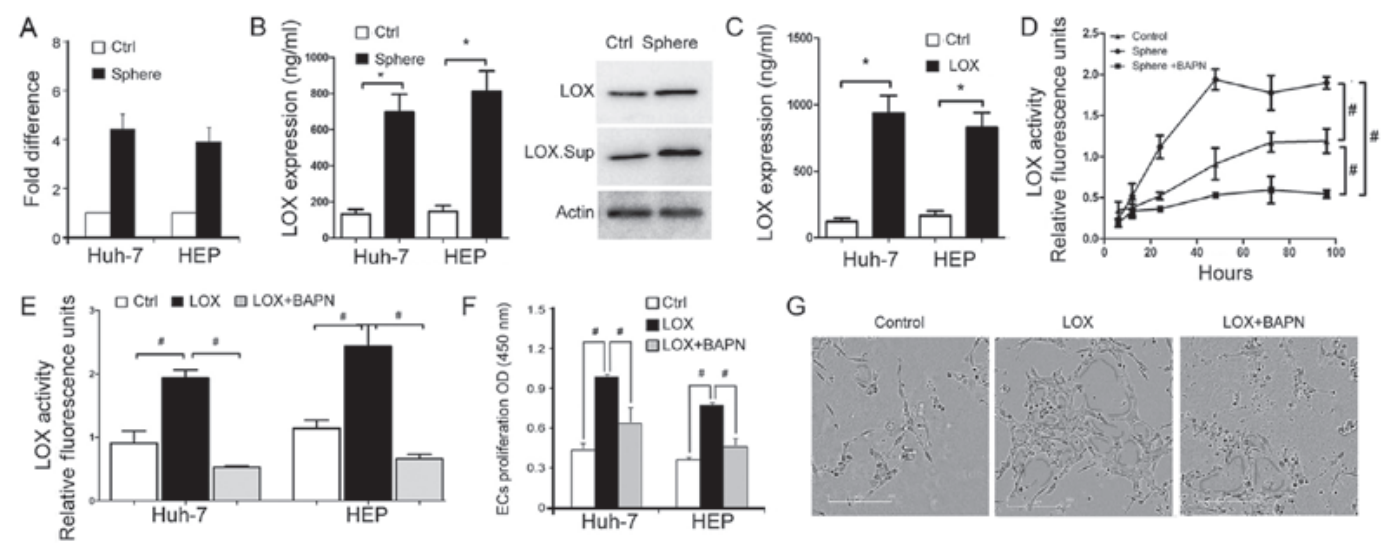

G

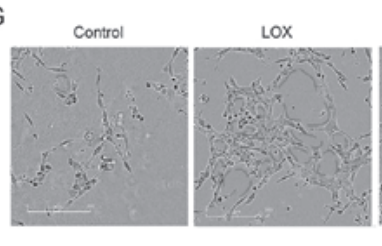

LOX+BAPN
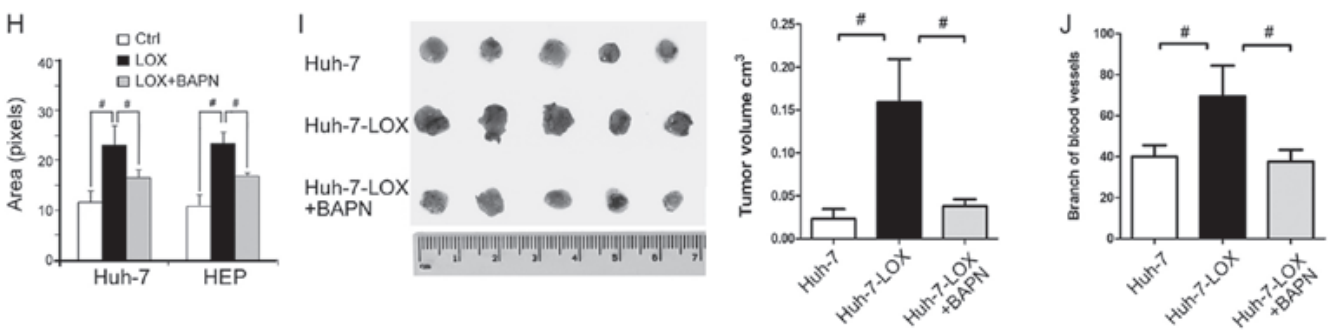

Figure 4. Sphere-derived cells enhance EC proliferation and tube formation through secretion of LOX. (A) Reverse transcription-quantitative polymerase chain reaction analysis of LOX mRNA levels relative to GAPDH mRNA levels in parental and spheres cell lines. (B) ELISA examination of LOX secretion in culture media of sphere-derived HuH-7 cells $(n=6)$ and western blot was performed to identify the LOX expression in cellular and extracellular-secreted in the supernatant. (C) LOX secretion from HEP and HuH-7 parental cells and genetic cells were determined by ELISA. (D) LOX activity was measured using the Fluorimetric Lysyl Oxidase Assay Kit Activity from sphere cells was significantly increased by LOX and arrested by BAPN (500 $\mu$ M) after $24 \mathrm{~h}$. (E) LOX overexpression cells enhanced LOX activity, which was downregulated by BAPN at $48 \mathrm{~h}$ post-treatment. (F) CM of parental cells and sphere-derived cells with or without BAPN treatment was collected. EC proliferation was examined by MTS using different CMs (n=9). (G) The capillary-like morphology of ECs cultured in HuH-7 medium is displayed, and $(\mathrm{H})$ the area is measured using ImageJ software. (I) HuH-7 and LOX genetic cells were transplanted onto chick chorioallantoic membrane, and tumor proliferation was measured following an 18-day incubation. The volume of the tumor [V = the length $\mathrm{x}$ (the width $x$ 2)/2] was highly reduced in the BAPN group. (J) Branching of vessels was statistically counted by ImageJ software. "P<0.05 using a two-sided Student's t-test; " $\mathrm{P}<0.05$ using one-way analysis of variance. CM, conditioned media; EC, endothelial cell; Ctrl, control; LOX, lysyl oxidase; Sup., supernatant; BAPN, $\beta$-aminopropionitrile.

was increased in HuH-7 and HEP cells overexpressing LOX (Fig. 4C). BAPN, a LOX inhibitor, binds to the active site of LOX. BAPN was used to inhibit the catalytic activity of LOX in sphere cells and LOX-overexpressing cells (Fig. 4D and E). The inhibition was detected using a fluorometric lysyl oxidase assay kit used to measure the release of active LOX from cells. 
A
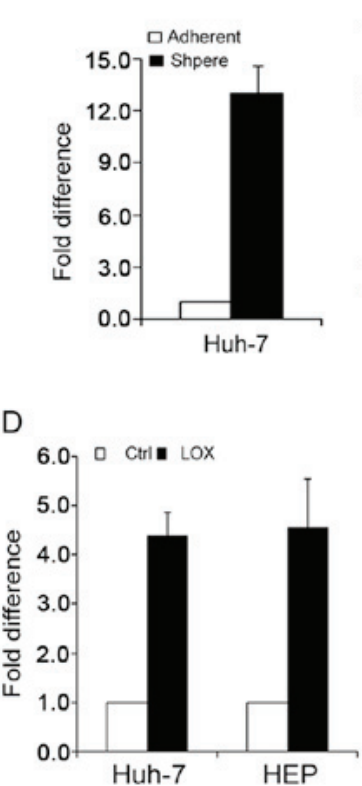

B VEGF
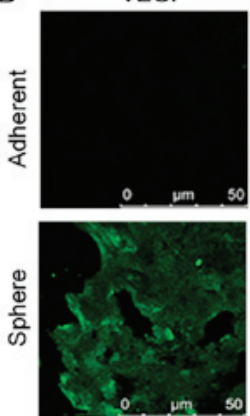

E
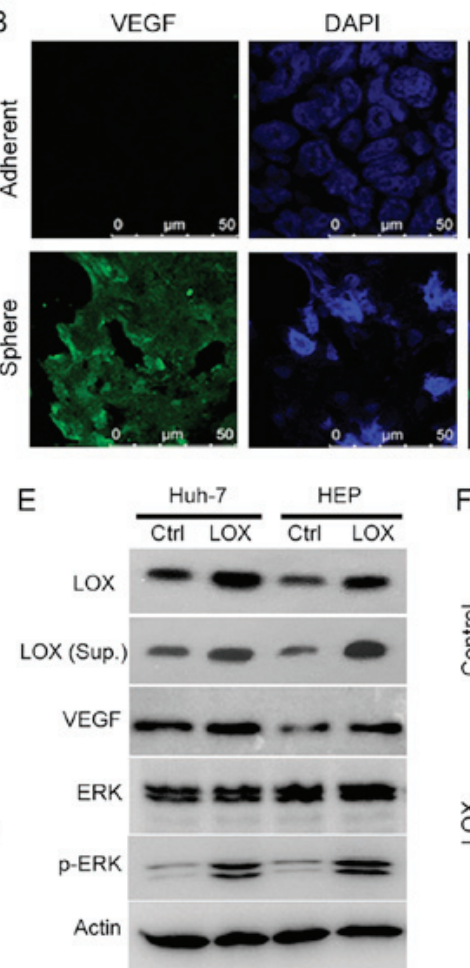

Overlay

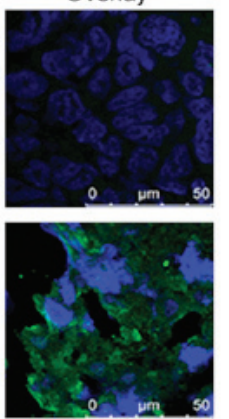

F
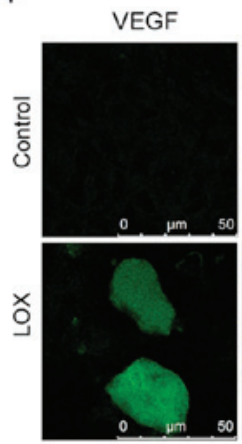

C

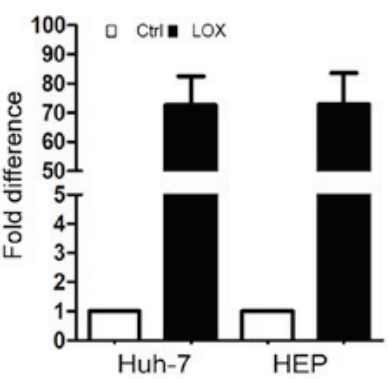

DAPI
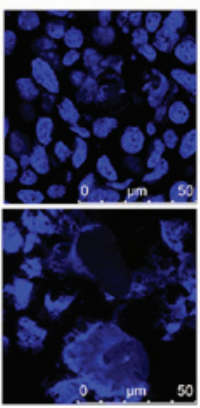

Overlay
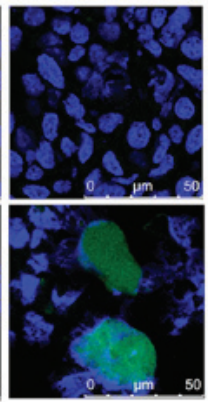

Figure 5. Spheres drive VEGF secretion by LOX. (A) VEGF mRNA level in parental and sphere cells. (B) Immunofluorescence staining of VEGF in parental and sphere cells. Nuclei were stained with DAPI. mRNA level of (C) LOX and (D) VEGF is identified in LOX genetic cells by reverse transcription-quantitative polymerase chain reaction. (E) Western blot analysis of LOX, VEGF and p-ERK protein expression. (F) Validation by immunofluorescence staining. VEGF, vascular endothelial growth factor; LOX, lysyl oxidase; Sup., supernatant.

\section{A}

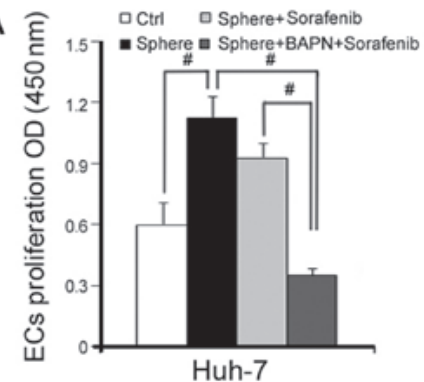

C

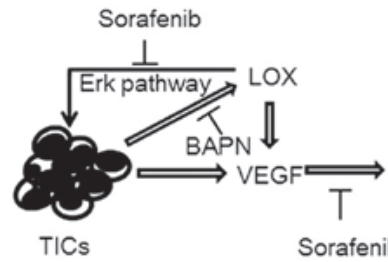

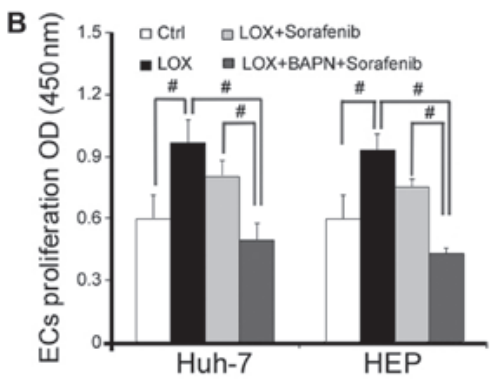

Proliferation

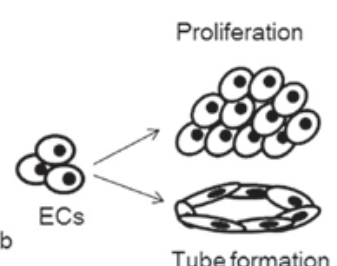

Figure 6. Therapeutic effects of a LOX inhibitor combined with sorafenib on EC proliferation. (A) EC proliferation was enhanced by CM from sphere cells and rescued by Sorafenib alone or in combination with BAPN. (B) Additional EC proliferation was enhanced by CM from LOX-induced cells or rescued by Sorafenib alone or in combination with BAPN. (C) Model depicting the proposed sphere-derived cellular mediated tumor angiogenesis via LOX secretion. LOX secretion is stimulated during the growth of sphere-derived cells, resulting in upregulation of VEGF expression, which strongly promotes EC proliferation and tube formation. This mechanism would be supported both by sorafenib and LOX inhibitor BAPN. ${ }^{~} \mathrm{P}<0.05$ using one-way analysis of variance. EC, endothelial cell; OD, optical density; BAPN, $\beta$-aminopropionitrile; LOX, lysyl oxidase; ERK, extracellular signal-regulated kinase; TICs, tumor-initiating cells; VEGF, vascular endothelial growth factor.

These data indicate that LOX secretion and activity may be important in TIC-enriched HCC cells.

TIC-enriched cells enhance EC proliferation and tube formation via LOX. Proliferation and tube formation of ECs was performed to identify whether TIC-enriched cells mediate angiogenesis via effects on LOX. CM of LOX-overexpressing cells significantly promoted EC viability compared with the control, while BAPN reversed this effect (Fig. 4F). The area of capillary-like structures in LOX-overexpressing cells was 
larger than in the control cell group, and BAPN suppressed EC tube formation (Fig. 4G and H). Furthermore, a CAM assay was used to assess tumor formation and the intensity of microvessels around tumor sites. BAPN significantly reduced the volume of LOX-HuH-7 tumors on the CAM and reduced the branching of microvessels that supported tumor proliferation (Fig. 4I and J). These data suggest that sphere-derived cells regulate EC proliferation and tube formation via LOX secretion and activity.

LOX stimulates TIC angiogenesis via VEGF expression. The findings of the current study have demonstrated that TIC-enriched cells enhance angiogenesis by secreting LOX. The expression of pro-angiogenesis factors at the molecular level and the response to tumor angiogenesis was investigated. As VEGF has an essential role in tumor angiogenesis, RT-qPCR and western blot analyses were performed to detect VEGF expression, comparing sphere-derived cells and parental control cells. The VEGF mRNA level was significantly upregulated in sphere cells compared with adherent cells (Fig. 5A). Similar results were validated using immunofluorescence staining (Fig. 5B). Furthermore, cells expressing LOX (Fig. 5C) were assessed to detect mRNA and protein levels of VEGF, ERK and p-ERK (Fig. 5D and E) and were validated using an immunofluorescence assay (Fig. 5F). It was demonstrated that LOX overexpression enhances VEGF expression in TIC-enriched cells.

Combination treatment of LOX inhibitor and sorafenib inhibits EC proliferation. Sorafenib is a drug widely used to treat HCC tumor angiogenesis (27). The therapeutic effects of sorafenib combined with the LOX inhibitor revealed that the functional behavior of angiogenesis is associated with ECs via cell proliferation. EC proliferation was enhanced by $\mathrm{CM}$ from HEP-sph and rescued by sorafenib or combination with BAPN (Fig. 6A). The same response was also observed in cells overexpressing LOX (Fig. 6B). These data suggest that ECs were suppressed by sorafenib, and that BAPN may improve the inhibition of sorafenib and exhibit an anti-angiogenesis function when LOX is overexpressed.

\section{Discussion}

Angiogenesis is the process by which new capillaries sprout or split from pre-existing vessels through the activation, proliferation, migration and channelization of ECs (43). Angiogenesis has an essential role in tumor growth and metastasis as newly formed capillaries are required to provide nutrients and remove waste from tumor tissues (44). Targeting tumor blood vessel growth can suppress neoangiogenesis and temporarily normalize tumor vessel structure, which may enhance tumor sensitivity to anti-cancer therapy. Multifactorial contributions of angiogenesis have been reported for decades, and the association between angiogenesis and matrix-modifying enzymes (45), which are required for remodeling of the extracellular matrix (ECM), is a new topic in cancer research. LOX is a collagen cross-linking enzyme, which has an essential role in creating a niche permissive for tumorigenesis in remodeling the vascular extracellular matrix during angiogenesis (46). The current study demonstrated that LOX was highly expressed in TICs and regulates the level of VEGF. Thus, there may be a link between TICs and angiogenesis mediated by LOX secretion and VEGF response in hUVECs (Fig. 6C).

As it has been demonstrated that sphere-derived tumor cells are TIC-enriched, two types of sphere-derived cells, HEP-sph and $\mathrm{HuH} 7$-sph, were generated. Gene and protein expression in liver cancer cells was analyzed, and it was demonstrated that stem-associated gene expression, including OCT4, Nanog, SOX2 and KLF4, were increased in the sphere cells than in the parental adherent cells. By analyzing bioinformatics data from multiple databases, LOX was identified as a candidate that is upregulated in TIC-enriched cells and downregulated in non-TIC cells. Therefore, the CM of cultured sphere-derived cells and LOX-overexpressing cells was collected to investigate the effect of TICs on hUVECs.

The current study revealed that LOX has a critical role in EC proliferation and contributes to angiogenesis in HCC, which can be induced by TIC enrichment. The evidence indicated that capillary vessels were observed adjacent to tumor tissues produced from sphere-derived cells. Additionally, GEO analysis and ELISA assays demonstrated that LOX was associated with TIC and VEGF. These results illustrated that LOX may be involved in mediating TIC-inducing angiogenesis. Thus, the tumor cell response to angiogenesis in cells overexpressing LOX was evaluated. Overexpressing LOX in cells promoted EC viability and tube formation, which indicated that LOX could be important for angiogenesis induced by TICs. Furthermore, a LOX inhibitor attenuated the effectiveness of sphere-derived cell CM on ECs, demonstrating that EC behaviors are inhibited by BAPN. By contrast, LOX increased ERK phosphorylation to promote HCC progression (33). Recently, Nareshkumar et al (47) reported that the pro-peptide domain of $\operatorname{LOX}(2.5 \mu \mathrm{g} / \mathrm{ml})$ repressed angiogenesis in hUVECs by reducing the phosphorylation status of focal adhesion kinase and ERK. These results led the current study to investigate the mechanism of how TIC affects angiogenesis via LOX. Immunofluorescence demonstrated that VEGF expression levels were high in sphere-derived cells, which represented neovascularization in these tissues. Finally, BAPN increased the anti-angiogenesis effects of sorafenib on EC viability and tube formation.

In summary, the findings of the present study indicate that as an ECM remodeling enzyme secreted by HCC TICs, LOX may promote angiogenesis by regulating VEGF expression. Although the function and mechanism of LOX in endothelial cells requires further investigation, these observations provide evidence that TICs promote tumor angiogenesis via LOX overexpression, and that a LOX inhibitor in combination with sorafenib may be a strategy for HCC therapy focused on angiogenesis.

\section{Acknowledgements}

Thanks are given to Dr Bin Dong (Department of Central Laboratory, Peking University Cancer Hospital and Institute, Beijing, China) for providing help with H\&E staining.

\section{Funding}

This study was supported by grants from the ' 863 ' Project (grant nos. 2014AA021606 and 2015AA020403); National 
Natural Science Foundation of China (grant nos. 81372594 and 81460360 and 81872025); Beijing Natural Science Foundation (grant no. 7182030); Natural Science Foundation of Xinjiang Uygur Autonomous Region (grant no. 2015211C126); Capital Funds for Health Improvement and Research 2018-2-1022.

\section{Availability of data and materials}

The datasets used and/or analyzed during the current study are available from the corresponding author on reasonable request.

\section{Authors' contributions}

WZ and BW supervised this study. MY, JL and ZL conceived the experiments and analyzed the data. BW analyzed data using the public database. MY, JL, FW, ZT and BM performed experiments, and MY, JL and WZ wrote the manuscript.

\section{Ethics approval and consent to participate}

All studies involving human samples adhered to the principles of the Declaration of Helsinki in accordance with the National Institutes of Health guidelines of patient consent. The acquisition and use of HCC tissue was approved by the Ethics Committee of PUCH. All animal experiments were approved by and conformed to the regulatory standards of Peking University Cancer Hospital on Laboratory Animals Care and Use in accordance with the National Institutes of Health Guide (Guide for the Care and Use of Laboratory Animals) (38).

\section{Patient consent for publication}

Not applicable.

\section{Competing interests}

The authors declare that they have no competing interests.

\section{References}

1. Chen W, Sun K, Zheng R, Zeng H, Zhang S, Xia C, Yang Z, Li H, Zou X and He J: Cancer incidence and mortality in China, 2014 Chin J Cancer Res 30: 1-12, 2018.

2. Forner A, Gilabert M, Bruix J and Raoul JL: Treatment of intermediate-stage hepatocellular carcinoma. Nat Rev Clin Oncol 11: 525-535, 2014.

3. Plaks V, Kong N and Werb Z: The cancer stem cell niche: How essential is the niche in regulating stemness of tumor cells? Cell Stem Cell 16: 225-238, 2015.

4. Zhao W, Wang L, Han H, Jin K, Lin N, Guo T, Chen Y, Cheng H, Lu F, Fang W, et al: 1B50-1, a mAb raised against recurrent tumor cells, targets liver tumor-initiating cells by binding to the calcium channel $\alpha 2 \delta 1$ subunit. Cancer Cell 23: 541-556, 2013.

5. Markowska A, Sajdak S, Markowska J and Huczynski A: Angiogenesis and cancer stem cells: New perspectives on therapy of ovarian cancer. Eur J Med Chem 142: 87-94, 2017.

6. Zimmerer RM, Ludwig N, Kampmann A, Bittermann G, Spalthoff S, Jungheim M, Gellrich NC and Tavassol F: CD24+ tumor-initiating cells from oral squamous cell carcinoma induce initial angiogenesis in vivo. Microvasc Res 112: 101-108, 2017.

7. Zheng YB, Gong JH, Liu XJ,Li Y and Zhen YS: A CD13-targeting peptide integrated protein inhibits human liver cancer growth by killing cancer stem cells and suppressing angiogenesis. Mol Carcinog 56: 1395-1404, 2017.
8. Kumar D, Kumar S, Gorain M, Tomar D, Patil HS, Radharani NNV, Kumar TVS, Patil TV, Thulasiram HV and Kundu GC: Notch1-MAPK Signaling Axis Regulates CD133+ Cancer Stem Cell-Mediated Melanoma Growth and Angiogenesis. J Invest Dermatol 136: 2462-2474, 2016.

9. Yi SY, Ruan J, Zhao L, Ke Y and Li XN: Metronomic gemcitabine targeted tumor vascular microenvironment decreases the population of CD133(+) cells in hepatocarcinoma xenografts. Cancer Biomark 14: 427-433, 2014.

10. Maehara O, Sato F, Natsuizaka M, Asano A, Kubota Y, Itoh J, Tsunematsu S, Terashita K, Tsukuda Y, Nakai M, et al: A pivotal role of Krüppel-like factor 5 in regulation of cancer stem-like cells in hepatocellular carcinoma. Cancer Biol Ther 16: 1453-1461, 2015.

11. Richtig G, Aigelsreiter A, Schwarzenbacher D, Ress AL, Adiprasito JB, Stiegelbauer V, Hoefler G, Schauer S, Kiesslich T, Kornprat $\mathrm{P}$, et al: SOX9 is a proliferation and stem cell factor in hepatocellular carcinoma and possess widespread prognostic significance in different cancer types. PLoS One 12: e0187814, 2017.

12. Zou S, Wang C, Liu J, Wang Q, Zhang D, Zhu S, Xu S, Kang M and He S: Sox12 Is a Cancer Stem-Like Cell Marker in Hepatocellular Carcinoma. Mol Cells 40: 847-854, 2017.

13. Mirshahidi S, Simental A, Lee SC, De Andrade Filho PA, Peterson NR, Cao W, Necochea-Campion R, Yang H, Duerksen-Hughes P and Yuan X: Subpopulations of cancer stem cells found in papillary thyroid carcinoma. Exp Cell Res 362: 515-524, 2018.

14. Jin B, Wang W, Meng XX, Du G, Li J, Zhang SZ, Zhou BH and Fu ZH: Let-7 inhibits self-renewal of hepatocellular cancer stem-like cells through regulating the epithelial-mesenchymal transition and the Wnt signaling pathway. BMC Cancer 16: 863, 2016.

15. Park IS, Chung PS and Ahn JC: Adipose-derived stem cell spheroid treated with low-level light irradiation accelerates spontaneous angiogenesis in mouse model of hindlimb ischemia. Cytotherapy 19: 1070-1078, 2017.

16. Ribeiro AL, Kaid C, Silva PBG, Cortez BA and Okamoto OK: Inhibition of Lysyl Oxidases Impairs Migration and Angiogenic Properties of Tumor-Associated Pericytes. Stem Cells Int 2017: 4972078, 2017.

17. Xie Q, Xie J, Tian T, Ma Q, Zhang Q, Zhu B and Cai X: Hypoxia triggers angiogenesis by increasing expression of LOX genes in 3-D culture of ASCs and ECs. Exp Cell Res 352: 157-163, 2017.

18. Bae WJ, Yi JK, Park J, Kang SK, Jang JH and Kim EC: Lysyl oxidase-mediated VEGF-induced differentiation and angiogenesis in human dental pulp cells. Int Endod J 51: 335-346, 2018.

19. Zhu J, Huang S, Wu G, Huang C, Li X, Chen Z, Zhao L and Zhao Y: Lysyl Oxidase Is Predictive of Unfavorable Outcomes and Essential for Regulation of Vascular Endothelial Growth Factor in Hepatocellular Carcinoma. Dig Dis Sci 60: 3019-3031, 2015.

20. Tse AP, Sze KM, Shea QT, Chiu EY, Tsang FH, Chiu DK, Zhang MS, Lee D, Xu IM, Chan CY, et al: Hepatitis transactivator protein $X$ promotes extracellular matrix modification through HIF/LOX pathway in liver cancer. Oncogenesis 7: 44, 2018.

21. Gao Y, Xiao Q, Ma H, Li L, Liu J, Feng Y, Fang Z, Wu J, Han X, Zhang J, et al: LKB1 inhibits lung cancer progression through lysyl oxidase and extracellular matrix remodeling. Proc Natl Acad Sci USA 107: 18892-18897, 2010.

22. Pickup MW, Laklai H, Acerbi I, Owens P, Gorska AE, Chytil A, Aakre M, Weaver VM and Moses HL: Stromally derived lysyl oxidase promotes metastasis of transforming growth factor- $\beta$-deficient mouse mammary carcinomas. Cancer Res 73: 5336-5346, 2013.

23. Kucharzewska P, Christianson HC, Welch JE, Svensson KJ, Fredlund E, Ringnér M, Mörgelin M, Bourseau-Guilmain E, Bengzon $\mathrm{J}$ and Belting $\mathrm{M}$ : Exosomes reflect the hypoxic status of glioma cells and mediate hypoxia-dependent activation of vascular cells during tumor development. Proc Natl Acad Sci USA 110: 7312-7317, 2013.

24. Matsuura S, Mi R, Koupenova M,Eliades A,Patterson S, Toselli P, Thon J, Italiano JE Jr, Trackman PC, Papadantonakis N, et al: Lysyl oxidase is associated with increased thrombosis and platelet reactivity. Blood 127: 1493-1501, 2016.

25. Pietras A, Katz AM, Ekström EJ, Wee B, Halliday JJ, Pitter KL, Werbeck JL, Amankulor NM, Huse JT and Holland EC: Osteopontin-CD44 signaling in the glioma perivascular niche enhances cancer stem cell phenotypes and promotes aggressive tumor growth. Cell Stem Cell 14: 357-369, 2014. 
26. Berretta M, Rinaldi L, Di Benedetto F, Lleshi A, De Re V, Facchini G, De Paoli P and Di Francia R: Angiogenesis Inhibitors for the Treatment of Hepatocellular Carcinoma. Front Pharmacol 7: 428, 2016

27. Montella L, Addeo R, Caraglia M and Del Prete S: Latest developments in targeted therapy for hepatocellular carcinoma. Expert Rev Anticancer Ther 10: 1635-1646, 2010.

28. Chen Y, Liu YC, Sung YC, Ramjiawan RR, Lin TT, Chang CC, Jeng KS, Chang CF, Liu CH, Gao DY, et al: Overcoming sorafenib evasion in hepatocellular carcinoma using CXCR4-targeted nanoparticles to co-deliver MEK-inhibitors. Sci Rep 7: 44123, 2017.

29. Gao JJ, Shi ZY, Xia JF, Inagaki Y and Tang W: Sorafenib-based combined molecule targeting in treatment of hepatocellular carcinoma. World J Gastroenterol 21: 12059-12070, 2015.

30. Trojan J and Waidmann O: Role of regorafenib as second-line therapy and landscape of investigational treatment options in advanced hepatocellular carcinoma. J Hepatocell Carcinoma 3 : $31-36,2016$.

31. Xu XL, Xing BC, Han HB, Zhao W, Hu MH, Xu ZL, Li JY, Xie Y, Gu J, Wang Y, et al: The properties of tumor-initiating cells from a hepatocellular carcinoma patient's primary and recurrent tumor. Carcinogenesis 31: 167-174, 2010

32. Su B, Zhao W, Shi B, Zhang Z, Yu X, Xie F, Guo Z, Zhang X, Liu J, Shen Q, et al: Let-7d suppresses growth, metastasis, and tumor macrophage infiltration in renal cell carcinoma by targeting COL3A1 and CCL7. Mol Cancer 13: 206, 2014.

33. Livak KJ and Schmittgen TD: Analysis of relative gene expression data using real-time quantitative PCR and the 2(-Delta Delta C(T)) method. Methods 25: 402-408, 2001.

34. Liu C, Guo C, Wang W, Zhu P, Li W, Mi Y, Myatt L and Sun K: Inhibition of Lysyl Oxidase by Cortisol Regeneration in Human Amnion: Implications for Rupture of Fetal Membranes. Endocrinology 157: 4055-4065, 2016.

35. Cheng T, Sun X, Wu J, Wang M, Eisenberg RA and Chen Z: Increased serum levels of tumor necrosis factor receptor-associated factor 1 (TRAF1) correlate with disease activity and autoantibodies in rheumatoid arthritis. Clin Chim Acta 462: 103-106, 2016.

36. Shu Q, Li W, Li H and Sun G: Vasostatin inhibits VEGF-induced endothelial cell proliferation, tube formation and induces cell apoptosis under oxygen deprivation. Int J Mol Sci 15: 6019-6030, 2014.

37. Giacomini A, Ackermann M, Belleri M, Coltrini D, Nico B, Ribatti D, Konerding MA, Presta M and Righi M: Brain angioarchitecture and intussusceptive microvascular growth in a murine model of Krabbe disease. Angiogenesis 18: 499-510, 2015.
38. National Research Council (US) Committee for the Update of the Guide for the Care and Use of Laboratory Animals: Guide for the Care and Use of Laboratory Animals. 8th edition. National Academies Press (US), Washington, DC, 2011

39. Pathan M, Keerthikumar S, Chisanga D, Alessandro R, Ang CS, Askenase P, Batagov AO, Benito-Martin A, Camussi G, Clayton A, et al: A novel community driven software for functional enrichment analysis of extracellular vesicles data. J Extracell Vesicles 6: 1321455, 2017.

40. Chandrashekar DS, Bashel B, Balasubramanya SAH, Creighton CJ, Ponce-Rodriguez I, Chakravarthi BVSK and Varambally S: UALCAN: A Portal for Facilitating Tumor Subgroup Gene Expression and Survival Analyses. Neoplasia 19: 649-658, 2017

41. Hodo Y, Honda M, Tanaka A, Nomura Y, Arai K, Yamashita T, Sakai Y, Yamashita T, Mizukoshi E, Sakai A et al: Association of interleukin-28B genotype and hepatocellular carcinoma recurrence in patients with chronic hepatitis C. Clin Cancer Res 19: 1827-1837, 2013

42. Osawa T, Ohga N, Akiyama K, Hida Y, Kitayama K, Kawamoto T, Yamamoto K, Maishi N, Kondoh M, Onodera Y, et al: Lysyl oxidase secreted by tumour endothelial cells promotes angiogenesis and metastasis. Br J Cancer 109: 2237-2247, 2013.

43. Liu J, Guo W, Xu B, Ran F, Chu M, Fu H and Cui J: Angiogenesis inhibition and cell cycle arrest induced by treatment with Pseudolarix acid B alone or combined with 5-fluorouracil. Acta Biochim Biophys Sin (Shanghai) 44: 490-502, 2012.

44. Bisacchi D, Benelli R, Vanzetto C, Ferrari N, Tosetti F and Albini A: Anti-angiogenesis and angioprevention: Mechanisms, problems and perspectives. Cancer Detect Prev 27: 229-238, 2003.

45. Li M, Li M, Yin T, Shi H, Wen Y, Zhang B, Chen M, Xu G, Ren K and Wei Y: Targeting of cancer associated fibroblasts enhances the efficacy of cancer chemotherapy by regulating the tumor microenvironment. Mol Med Rep 13: 2476-2484, 2016.

46. Semenza GL: Cancer-stromal cell interactions mediated by hypoxia-inducible factors promote angiogenesis, lymphangiogenesis, and metastasis. Oncogene 32: 4057-4063, 2013.

47. Nareshkumar RN, Sulochana KN and Coral K: Inhibition of angiogenesis in endothelial cells by Human Lysyl oxidase propeptide. Sci Rep 8: 10426, 2018. 